\title{
Estudio sonográfico del diafragma. Una nueva herramienta diagnostica para el anestesiólogo
}

\section{Diafragmatic Ultrasound, a new tool for the anesthesiologist}

CECILIA M. ACOSTA ${ }^{1}$, URBANO M.S. ${ }^{2}$, TUSMAN G. ${ }^{1}$.

\begin{abstract}
Point-of-care ultrasonography has become a widely used diagnostic tool in the intensive care units and during perioperative settings. Nowadays, ultrasound has been also employed to evaluate diaphragmatic function. Some advantages of this method include safety, absence of ionizing radiation, and availability of real-time bedside examinations. The aim of this review is to promote the use of diaphragmatic ultrasound assessment among anesthesiologists and intensive care physicians. This article describes the standard diaphragmatic ultrasound technique and the knowledge required in order to monitor and diagnose diaphragmatic dysfunction; emphasizing its use in the operating room and in the different fields of clinical application.
\end{abstract}

\section{RESUMEN}

El ultrasonido point-of-care se ha convertido en una herramienta diagnóstica ampliamente utilizada en unidades de cuidados intensivos y durante el período peri-operatorio. En la actualidad, el ultrasonido esta siendo empleado además para evaluar la función diafragmática. Las ventajas de este método incluyen seguridad, ausencia de radiación ionizante y posibilidad de realizar examinación en
Key words: Ultrasound, diaphragmatic dysfunction, anesthesia, intensive care.

\section{Palabras clave:}

Ultrasonido, disfunción diafragmática, anestesia, cuidados intensivos.

Servicio de Anestesia, Hospital Privado de Comunidad, Mar del Plata, Buenos Aires, Argentina.

Servicio de Cuidados Intensivos, Clínica Pueyrredón, Mar del Plata, Buenos Aires, Argentina.

Fecha recepción: 10 de marzo de 2018

Fecha aceptación: 17 de marzo de 2018

\section{ORCID}

https://orcid.org/0000-0003-0063-6

\section{Correspondencia:}

Cecilia M. Acosta

Servicio de Anestesia.

Hospital Privado de Comunidad, Mar del Plata, Buenos Aires, Argentina.

Córdoba 4545, CP 7600, Mar del Plata, Buenos Aires, Argentina.

Teléfono: +54 2234990074

Fax: +54 2234990099

Email: ceacosta77@icloud.com 
tiempo real a la cabecera del paciente. El objetivo de esta revisión es promover el uso de la evaluación sonográfica del diafragma para médicos anestesiólogos e intensivistas. Este artículo describe la técnica estándar de la evaluación sonográfica del diafragma y el conocimiento requerido para el diagnóstico y monitorización de la disfunción diafragmática, enfatizando el uso en quirófano y en los diferentes campos de aplicación clínica.

\section{Introducción}

E I diafragma presenta una estructura muscular en forma de domo que separa la cavidad torácica de la abdominal, se encuentra compuesto por fibras tipo I de baja resistencia y tipo II de contracción rápida, y su inervación esta dada por el nervio frénico que se origina de las raíces C3-C5[1]. Su parte media es tendinosa y es denominada "centro frénico", desde donde parten fibras musculares radiadas que descienden hacia el esternón, las costillas y las vertebras lumbares. Debido a que los arcos costales anteriores son más altos que los posteriores, la inserción del diafragma es más alta en la parte anterior que posterior. Su inserción a nivel de la parte inferolateral de la caja torácica, es denominada "zona de aposición"[2]. Durante la inspiración el diafragma se contrae y provoca un desplazamiento caudal del mismo, lo que genera un aumento en la presión de la cavidad abdominal a nivel de la zona de aposición incrementando de esta manera el diámetro transverso del tórax. Constituye el principal músculo inspiratorio, siendo el responsable del incremento del $75 \%$ del volumen pulmonar durante una respiración normal, mientras que los músculos intercostales, escalenos y esternocleidomastoideo aportan el 25\% restante. En pacientes de alto riesgo su disfunción puede provocar fallo respiratorio asociado a hipercapnia y en casos mas severos ocasionar hipoxemia.

Dentro de las causas que generan disfunción diafragmática podemos mencionar la injuria del nervio frénico, post-operatorio de cirugía cardiaca, torácica o abdominal, asociada a bloqueo del nervio frénico durante el bloqueo del plexo braquial, enfermedades neuromusculares, metabólicas, infecciosas, disfunción diafragmática asociada al paciente crítico secundaria a polineuropatía, miopatía, drogas, etc. La ventilación mecánica puede inducir por si misma disfunción diafragmática, denominada "disfunción diafragmática inducida por el ventilador" debido a atrofia e injuria local. Existe una correlación directa entre la duración de la ventilación mecánica y el grado de atrofia muscular; por el contrario, períodos breves de actividad diafragmática durante la ventilación mecánica ya sea a través de ventilación espontánea intermitente o estimulación directa del nervio frénico limita tal atrofia. Por consiguiente, la estrategia de reducir el tiempo de ventilación controlada y promover la ventilación asistida podría ayudar a limitar la aparición de disfunción diafragmática asociada a la ventilación mecánica.

Es importante destacar que la disfunción diafragmática constituye uno de los factores determinantes en la patogénesis de las complicaciones pulmonares post-operatorias asociada a cirugías de tórax y abdomen superior. Por consiguiente, el deterioro en la reserva contráctil del diafragma puede prolongar la necesidad de asistencia ventilatoria mecánica, retrasar o conducir al fracaso del periodo de weaning y prolongar la estancia en cuidados intensivos.

\section{Evaluación sonográfica del diafragma}

Diferentes métodos permiten evaluar la función diafragmática: como la radiografía de tórax, tomografía, resonancia magnética dinámica y fluoroscopía; sin embargo, todos ellos implican el traslado del paciente critico fuera del área de cuidados intensivos, son imprácticos y algunos de ellos utilizan radiación.

La utilidad del ultrasonido (US) en modo B y M para el estudio cualitativo de la cinética diafragmática fue reportado por primera vez en el año 1975 por Harper, quien evidenció el movimiento de los órganos abdominales[3]. En los últimos años la evaluación sonográfica del diafragma ha ganado popularidad, debido a que permite valorar la función diafragmática a la cabecera del paciente, sin exponerlo a radiación ionizante y de manera rápida y no invasiva.

Se han descrito diversos métodos de exploración para evaluar la cinética diafragmática tanto de manera directa como indirecta con diferentes tipos de sondas. Las sondas de alta frecuencia 6-13 MHz son de elección para el estudio del engrosamiento diafragmático debido a que ofrecen mayor resolución en campos proximales, mientras que las sondas convex de 2-6 $\mathrm{MHz}$ o sectorial de 2-5 MHZ, al poseer baja frecuen- 


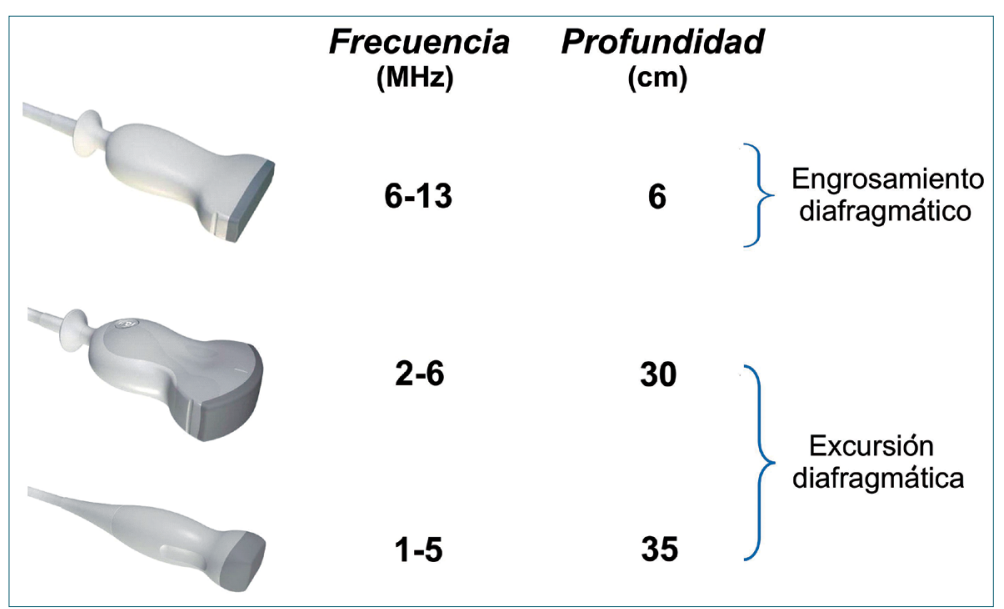

Figura 1. Sondas ecográficas empleadas para el estudio sonográfico del diafragma.
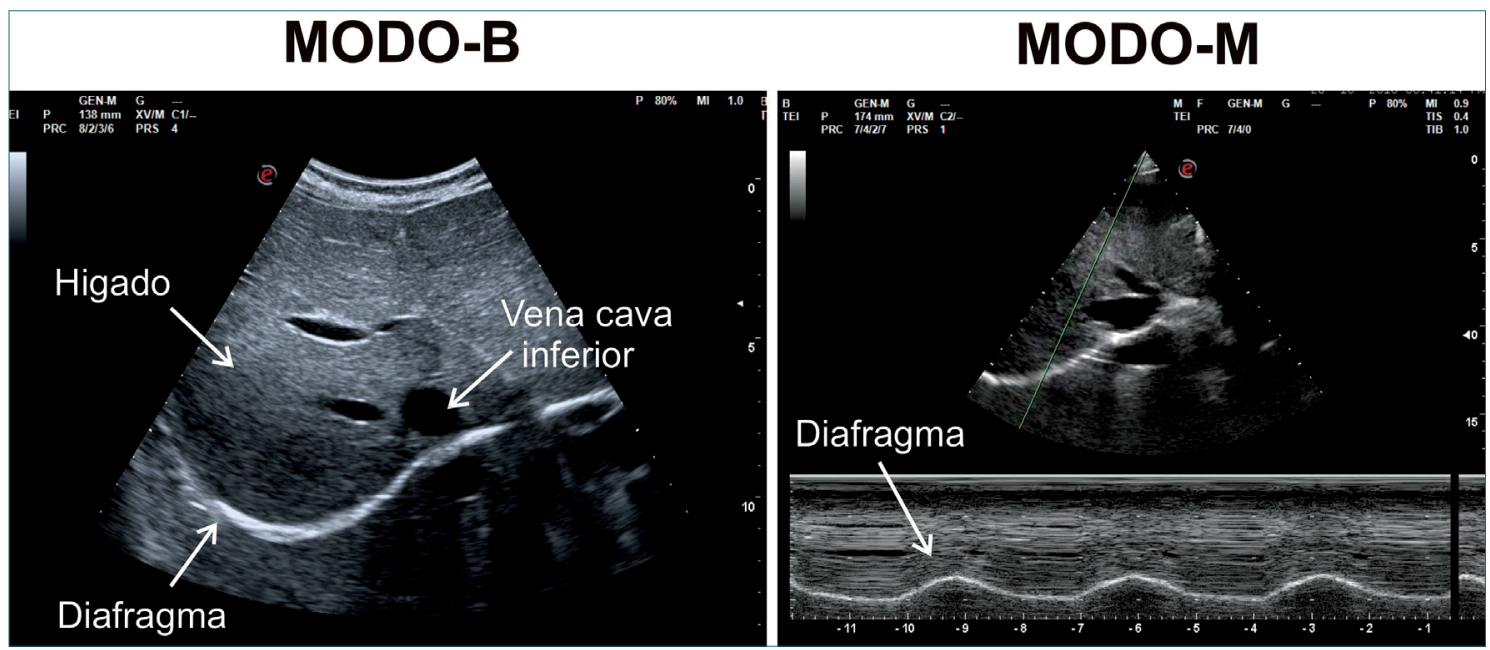

Figura 2. Modalidades ecográficas utilizadas para examinar la cinética diafragmática. A la derecha imagen obtenida usando la ventana acústica hepática, con sonda convex utilizando modo B; a la izquierda se observa el diafragma como una línea hiperecoica con movimiento sinusoidal, en modo M, con ascenso durante la inspiración y descenso en espiración.

cia permiten el estudio de la excursión diafragmática (Figura 1). Boussuges y colaboradores, reportaron que la reproducibilidad del método intra-observador es de 96 y 94\%; inter-observador de $95-91 \%$ durante ventilación espontánea para el hemidiafragma derecho e izquierdo respectivamente[4]. El US posee mayor certeza diagnostica en la evaluación de la función diafragmática que otros métodos portátiles como la fluoroscopía[5]; examina preferentemente la parte posterior y lateral del diafragma, la cual representa principalmente el componente muscular inervado por el nervio frénico, a diferencia de la fluoroscopía que estudia el área tendinosa anterior, conformada por el centro frénico que se moviliza un $40 \%$ menos con la respiración. La fluoroscopía posee además, como desventaja, la exposición repetida a radiación ionizante y la imposibilidad de evaluar pacientes con neumotórax o derrame pleural.

El estudio sonográfico de la función diafragmática se realiza con el paciente en posición supina, de esta manera podemos evaluar la cinética diafragmática utilizando tanto el modo B como M[3]-[6] (Vídeo 1) a través de dos metodologías diferentes (Figura 2 y 3):

- Movimiento diafragmático: examina la excursión diafragmática en tiempo real. (Vídeo 2).

- Morfología diafragmática: medición del engrosamiento del diafragma. (Vídeo 3). 


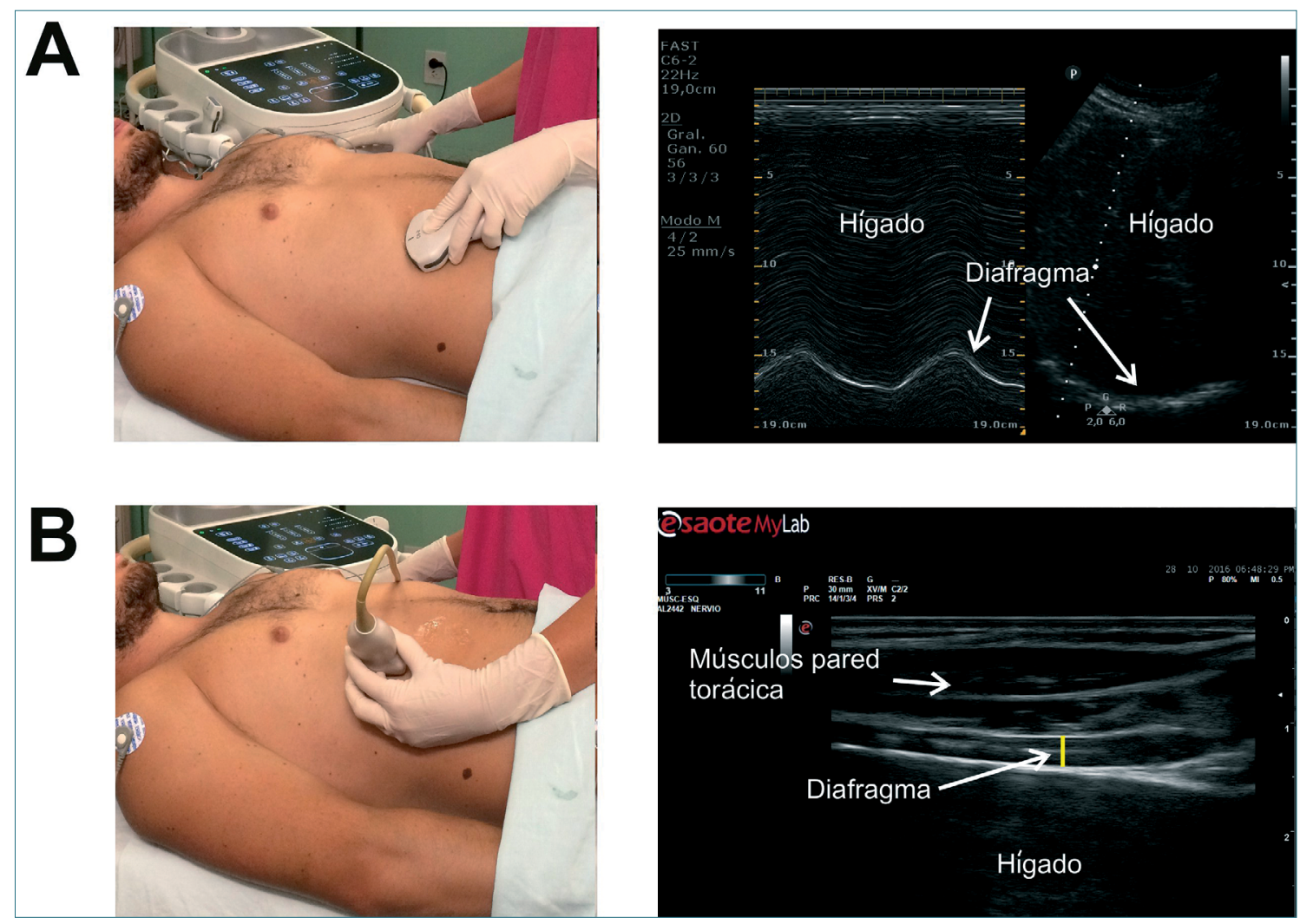

Figura 3. A: A la izquierda se observa posición de la sonda convex 2-6 $\mathrm{MHz}$ para escaneo del diafragma en el abordaje subcostal anterior a la altura de la línea medio-clavicular; a la derecha imagen ecográfica del diafragma en modo $\mathrm{M}$ y $\mathrm{B}$ examinando la excursión diafragmática. B: A la izquierda escaneo del diafragma en línea axilar anterior a nivel del $8^{\circ}-9^{\circ}$ espacio intercostal "zona de aposición" con sonda lineal 6-13 MHz; a la derecha imagen sonográfica en modo B para evaluar el engrosamiento diafragmático.

\section{A- Evaluación de la excursión diafragmática}

Para estudiar la excursión diafragmática utilizaremos una sonda convex 2-6 MHz o sectorial 2-5 MHz. Existen tres abordajes diferentes (Figura 4 y 5 ):

- Abordaje subcostal anterior: con el paciente en decúbito supino se posiciona la sonda a nivel subcostal a la altura de la línea axilar anterior o medio-clavicular, orientando la sonda en dirección cefálica y posterior. De esta forma el rayo de US alcanza la parte perpendicular del tercio posterior del hemidiafragma[4]-[5]. (Vídeo 4).

- Abordaje subcostal posterior: permite examinar el diafragma con el paciente sentado o en posición supina, presenta como limitante la imposibilidad de observar todo el diafragma cuando el pulmón se encuentra aireado en las bases debido al "signo de la cortina" (Vídeo 5).

- Abordaje subxifoideo: útil en pacientes delgados y en niños debido a que permite evaluar la excursión de ambos hemidiafragmas de manera simultánea. Con el paciente en decúbito supino se posiciona transversalmente la sonda convex debajo de la apófisis xifoides. (Vídeo 6).

Si utilizamos modo B observaremos una línea hiperecoica que representa al diafragma, que normalmente se aproximara a la sonda cuando el paciente inspira y se alejara en espiración, mientras que con el modo M observaremos un movimiento sinusoidal, con ascenso durante la inspiración y descenso en espiración.

Debido a la excelente ventana acústica que ofrece el hígado, el hemidiafragma derecho es posible de visualizar de manera mas fácil y constante, a diferencia del lado izquierdo, donde la menor ventana acústica del bazo, en algunas oportunidades, dificulta su examinación, al igual que el artefacto generado por el 
ABORDAJE SUBCOSTAL ANTERIOR

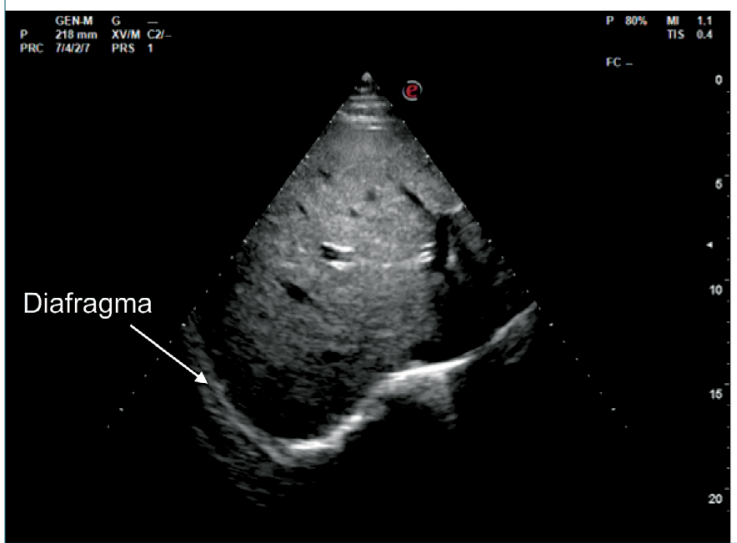

ABORDAJE SUBXIFOIDEO

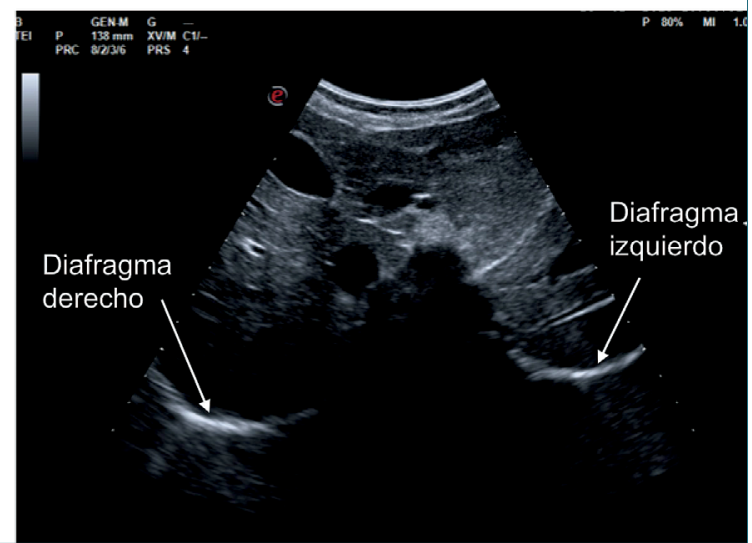

Figura 4. Principales ventanas sonográficos para el estudio del diafragma. A la izquierda imagen obtenida con sonda sectorial 2-5 MHz, utilizando la ventana subcostal a nivel de la línea medio-clavicular, se observa el hemidiafragma derecho en modo $\mathrm{M}$ y $\mathrm{B}$. A la derecha ventana subxifoidea utilizada en pacientes pediátricos, imagen obtenida con sonda convex 2-6 $\mathrm{MHz}$, se observa el hemidiafragma derecho e izquierdo en modo B.



Figura 5. Abordaje subcostal posterior: imágenes obtenidas con sonda convex 2-6 MHz a nivel de la línea axilar media. A la izquierda los artefactos generados por el pulmón aireado impiden visualizar el diafragma en toda su extensión. A la derecha la presencia de derrame pleural genera una excelente ventana acústica para observar completamente el diafragma.

aire en la cámara gástrica. La presencia de derrame pleural o consolidaciones como neumonías o atelectasias optimizan la ventana acústica a nivel del abordaje subcostal posterior y anterior, lo que permiten visualizar el diafragma en toda su extensión (Figura 5) (Vídeo 5 y 7).

Para evaluar la excursión diafragmática y evitar mediciones erróneas, es importante localizar la mejor imagen en modo B orientando el transductor hacia la parte posterior del diafragma para luego trazar el modo M lo más perpendicular posible a la parte posterior del mismo. La evaluación de la excursión se puede realizar en tres momentos: durante respiración tranquila, profunda y solicitando al paciente que realice la maniobra de sniff[4]. Esta última maniobra consiste en una inspiración voluntaria rápida por la nariz con la boca cerrada, permite evaluar la fuerza contráctil del diafragma y detectar rápidamente parálisis diafragmática cuando se observa un movimiento paradójico o ausencia de movimiento. El punto de corte para definir disfunción diafragmática es una excursión < $1 \mathrm{~cm}$ durante respiración tranquila[4]. La dirección del trazado en modo $\mathrm{M}$ en el hemidiafragma paralizado permite distinguir entre paresia o 
parálisis observando el movimiento caudal o craneal respectivamente. Por consiguiente, en aquellos pacientes que presentan paresia, se observará una reducción del movimiento hacia caudal en inspiración estará representado en el modo $\mathrm{M}$ como una onda por encima de la línea de base con una excursión muy limitada, mientras que en una parálisis el movimiento será paradojal observándose una inflexión por debajo de la línea de base en el modo M[7] (Figura 6). Sin embargo, existen casos en los cuales se ha reportado un movimiento paradojal del hemidiafragma a pesar de no existir parálisis diafragmática, como en el caso de fibrosis pulmonar, atelectasias, neumotórax con presión negativa, hidrotórax y absceso subfrénico[8]. Tabla 1.
Normalmente existe una asimetría en excursión máxima de ambos diafragmas, siendo mayor excursión en el hemidiafragma izquierdo y menor en el derecho; no obstante, la excursión del hemidiafragma derecho no debe ser inferior al 50\% del desplazamiento máximo del izquierdo.

Es importante tener presente que si el paciente se encuentra bajo asistencia ventilatoria, ya sea asistida o controlada, es necesario suspender momentáneamente la misma poder evaluar la función diafragmática real y evitar un sesgo en la medición, ya que de otra manera no es posible discriminar la fuerza generada por la contracción diafragmática del paciente y desplazamiento pasivo producido por la presión positiva externa.



Respiración tranquila Excursión diafragmática $1.5-2 \mathrm{~cm}$

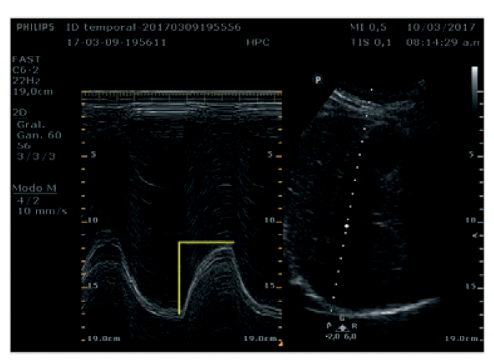

Respiración forzada Excursión diafragmática $6-7 \mathrm{~cm}$

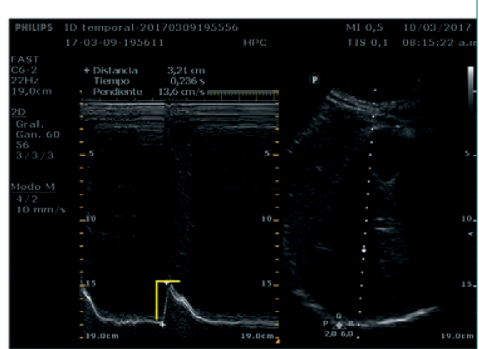

Maniobra sniffing Excursión diafragmática $2.5-3 \mathrm{~cm}$

Figura 6. Excursión diafragmática utilizando la sonda convex 2-6 $\mathrm{MHz}$ a nivel subcostal en línea medio-clavicular, en modo $\mathrm{M}$ y $B$, durante respiración tranquila, forzada y maniobra de sniffing.

\begin{tabular}{|c|c|c|c|c|c|c|}
\hline & Sonda & Abordaje & $\begin{array}{c}\text { Modo } \\
\text { examinación }\end{array}$ & $\begin{array}{l}\text { Respiración } \\
\text { normal }\end{array}$ & $\begin{array}{c}\text { Respiración } \\
\text { profunda }\end{array}$ & $\begin{array}{c}\text { Maniobra } \\
\text { sniff }\end{array}$ \\
\hline Excursión cm & $\begin{array}{l}\text { convex o } \\
\text { sectorial }\end{array}$ & subcostal & $B-M$ & $1,5-2$ & $6-9$ & $2,5-3$ \\
\hline $\begin{array}{l}\text { Engrosamiento } \\
\mathrm{mm}\end{array}$ & $\begin{array}{c}\text { lineal } 6 \text { - } 12 \\
\mathrm{MHz}\end{array}$ & $8^{\circ}-9^{\circ} \mathrm{EIC}$ & $B-M$ & 2,8 & $>4$ & \\
\hline FED \% & $\begin{array}{c}\text { lineal } 6 \text { - } 12 \\
\mathrm{MHz}\end{array}$ & $8^{\circ}-9^{\circ} \mathrm{EIC}$ & $B-M$ & $\geq 36 \%$ & $28-96$ & $>40$ \\
\hline Velocidad cm/s & $\begin{array}{l}\text { convex o } \\
\text { sectorial }\end{array}$ & subcostal & $M$ & $1,3 \pm 0,4$ & $>1,52$ & 10,4 \\
\hline
\end{tabular}

EIC: Espacio intercostal. FED: Fracción de engrosamiento diafragmático. 


\section{B- Evaluación del engrosamiento diafragmático}

Utilizaremos una sonda lineal de alta frecuencia 6-12 MHz.

- Abordaje intercostal: con el paciente en decúbito supino se posiciona la sonda en el $8^{\circ}-9^{\circ}$ espacio intercostal a nivel de la línea medio-axilar. Este nivel constituye la llamada "zona de aposición", ya que el diafragma se yuxtapone con la caja torácica[5]. Observaremos al diafragma como una estructura compuesta por tres capas: dos capas hiperecoicas corresponden a la pleura diafragmática y al peritoneo, entre medio de ambas el músculo diafragmático se observa como una estructura hipoecoica. Durante la inspiración a capacidad pulmonar total observaremos un incremento en el grosor diafragmático y una disminución del mismo al final de la espiración al alcanzar el volumen residual, utilizando tanto modo B o M. (Figura 7).

Recientemente Tsui y colaboradores reportaron una mnemotecnia "ABCDE" que ayuda a recordar el sitio donde debemos realizar el escaneo para la evaluación del diafragma en la ventana intercostal[9]. De esta manera debemos posicionar la sonda lineal de alta frecuencia en la línea Axilar anterior a nivel del $8^{\circ}-10^{\circ}$ espacio intercostal, donde identificaremos si el paciente respira (Breathing) a través del signo del "deslizamiento pulmonar" o "lung sliding" durante el ciclo respiratorio, a continuación desplazaremos la sonda Caudalmente hasta identificar el Diafragma para poder realizar su Evaluación.

En pacientes pediátricos, dada la pequeña dimensión del tórax, este abordaje permite evaluar en un mismo escaneo varios espacios intercostales y reconocer de manera simultanea el deslizamiento pleural y movimiento diafragmático[10].

El grosor diafragmático normal medido en la zona de aposición al final de una espiración normal es de 2,2-2,8 mm, durante una inspiración máxima puede alcanzar $4 \mathrm{~mm}$ o más[11], en tanto que valores $<2$ $\mathrm{mm}$ indican atrofia diafragmática. En pacientes que se encuentran bajo asistencia ventilatoria mecánica, el engrosamiento diafragmático es el indicador más confiable del esfuerzo respiratorio, ya que refleja la activación contráctil del diafragma más que el incremento del volumen torácico per se[12]; en tanto que la excursión no debería ser usada para evaluar cuantitativamente la actividad contráctil del diafragma[13].

Los cambios en el engrosamiento se evalúan a través de la fracción de engrosamiento diafragmática (FDE):
FED \% = Grosor al fin de inspiración - grosor al fin de espiración x 100 Grosor al fin de espiración

Utilizando el modo $M$ es posible medir la velocidad de contracción diafragmática a través de la ventana subcostal. La misma se calcula mediante el cociente entre la excusión diafragmática durante la inspiración y el tiempo inspiratorio y se expresa en $\mathrm{cm} /$ segundos (Figura 8). Durante una ventilación normal la velocidad de contracción diafragmática es de 1,5 $\mathrm{cm} / \mathrm{seg}$, mientras que valores $<1 \mathrm{~cm} / \mathrm{seg}$ son indicadores de disfunción diafragmática.

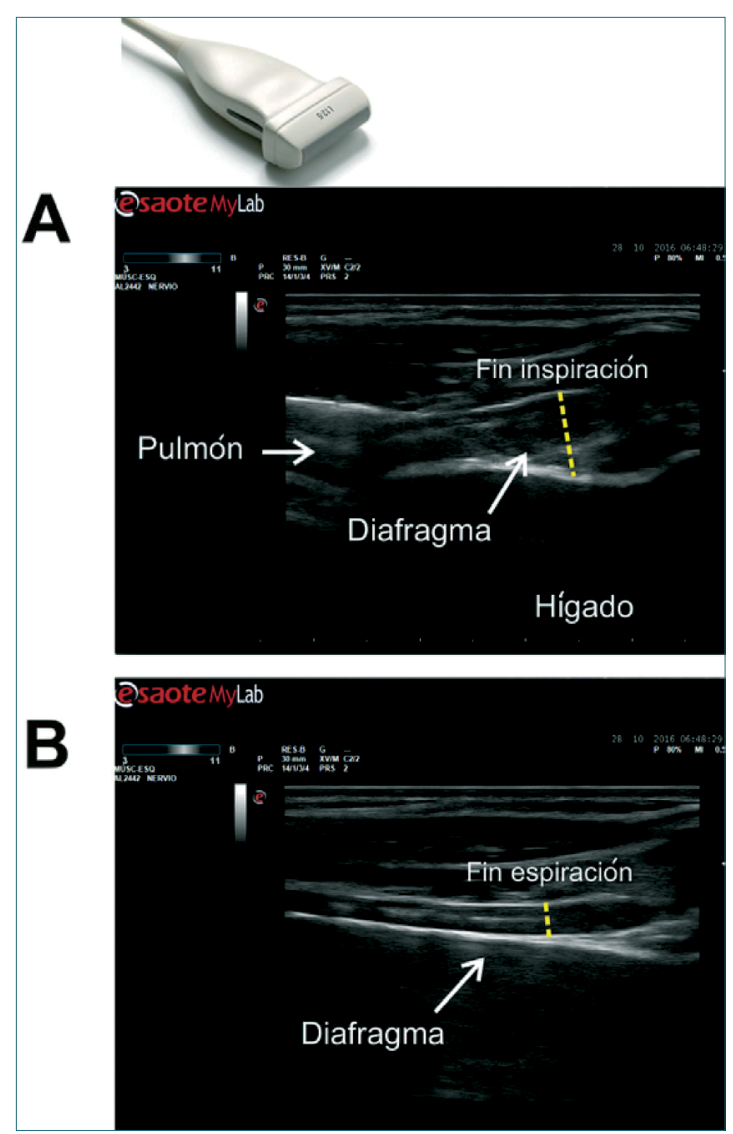

Figura 7. Evaluación del engrosamiento diafragmático utilizando sonda lineal 6-13 $\mathrm{MHz}$ a nivel de la "zona de aposición". Imágenes obtenidas en modo B. A: al final de la inspiración (máximo engrosamiento), B: al final de la espiración alcanzando el volumen residual (mínimo engrosamiento). 


\section{Aplicaciones clínicas}

\section{1- Paresia diafragmática debida a bloqueo del plexo braquial}

El bloqueo del plexo braquial, principalmente el abordaje interescalénico, se asocia a parálisis ipsilateral del hemidiafragma ipsilateral debido a la diseminación del anestésico local sobre el nervio frénico, el cual controla el movimiento diafragmático y su contracción. La incidencia de parálisis transitoria del nervio frénico es del $100 \%$ cuando se utilizan técnicas guiadas por reparos anatómicos y parestesias[14]-[15]. Dicha parálisis se encuentra limitada a la duración de acción del anestésico local y aparece cuando se utilizan volúmenes de anestésico local mayores a 10 ml[14]-[16]. Se ha reportado una incidencia del 45\% de parálisis diafragmática ipsilateral con volúmenes reducidos (5 ml) utilizando técnicas ecoguiadas[17].

Si bien es posible evidenciar con US la paresia diafragmática luego de 5 minutos de administrado el anestésico local, debería repetirse la examinación a los 15 y 30 minutos luego de realizado el bloqueo para dar tiempo al desarrollo completo de la parálisis del nervio frénico[14],[15]. En presencia de paresia diafragmática la presión inspiratoria es alcanzada en gran parte por la contracción de los músculos intercostales y la expansión de la caja torácica[18]. Numerosos estudios realizados en pacientes sanos con parálisis diafragmática unilateral han reportado que la saturación de oxígeno puede permanecer sin cambios o existir una disminución menor al 7\%[17],[19]-[20], probablemente debido a que la aparición de hipoxemia posee una etiología multifactorial. En contraste, existen datos que muestran que la aparición de una paresia o parálisis diafragmática podría ocasionar un fallo respiratorio agudo cuando el paciente presenta múltiples comorbilidades, recibe elevados volúmenes y/o concentración de anestésicos locales o existe disminución de la reserva contráctil en el hemidiafragma contralateral[21]-[23]. Altintas y su grupo reportaron episodios de saturación de oxígeno < $85 \%$ en el $10 \%$ en pacientes con insuficiencia renal crónica que recibieron bloqueo interescalénico con volúmenes de anestésico local de $30 \mathrm{ml}$ para realización de fístula arterio-venosa[23].

El examen sonográfico del tórax permite al anestesiólogo que realiza anestesia loco-regional evaluar y realizar el diagnóstico rápido de algunas de las complicaciones asociadas a esta técnica anestésica como la aparición de disnea aguda debida a neumotórax o disfunción diafragmática, principalmente en pacientes de alto riesgo[24].

Si bien el método de referencia para el diagnós- tico de parálisis bilateral es la medición de la presión transdiafragmática continua, es poco sensible para el diagnóstico de parálisis unilateral, partiendo del concepto que un hemidiafragma es suficiente para generar adecuada presión transdiafragmática durante respiración tranquila[5]. De igual manera, el US posee mayor sensibilidad que la saturación de oxígeno para evidenciar la disfunción diafragmática unilateral, debido a que ésta última evalúa la función pulmonar de manera bilateral, incluyendo el uso de los músculos accesorios y la actividad diafragmática contralateral. Al-Kaisy y su grupo estudiaron los efectos respiratorios en pacientes que recibieron bloqueo interescalénico con bajas dosis de bupivacaína, reportando que la aparición de hemiparesia diafragmática se asoció a una disminución de la capacidad vital forzada y del VEF, del 75 y $78 \%$, respectivamente; sin embargo, dichos pacientes permanecieron asintomáticos sin necesidad de recibir tratamiento[25]. A pesar de existir una clara correlación entre los cambios en los test de función pulmonar y la evidencia de paresia diafragmática a través de US, aun no existen estudios que evalúen específicamente la correlación entre el US, los test de función pulmonar, la saturación de oxígeno y los síntomas subjetivos de disnea[26].

La evaluación sonográfica del diafragma presenta una elevada sensibilidad (93\%) y especificidad (100\%) para el diagnóstico de disfunción del nervio frénico[27], y como hemos mencionado, posee mayor agudeza diagnóstica que otros métodos portátiles como la fluoroscopía[5],[28]-[30]. Constituye la técnica de imagen de elección para el anestesiólogo, ya que permite evaluar la función diafragmática de manera dinámica, rápida, no invasiva y a la cabecera del paciente. No solo es posible realizar el diagnóstico de disfunción diafragmática sino también evaluar la recuperación realizando escaneos repetidos a través del tiempo. Cuando ocurre parálisis diafragmática ipsilateral al bloqueo interescalénico la expansión pulmonar se ve limitada y puede ocurrir ausencia de movimiento o un movimiento paradojal del hemidiafragma paralizado en dirección cefálica durante la inspiración.

Como hemos descripto anteriormente existen dos métodos para realizar el estudio sonográfico de la función diafragmática. El primero consiste en medir la excursión diafragmática durante la inspiración con una sonda convex de 3-5 $\mathrm{MHz}$ en modo $\mathrm{B}$ o $\mathrm{M}$, utilizando la ventana acústica del hígado y el bazo (Vídeo 8); en ocasiones puede ser difícil de visualizar principalmente del lado izquierdo y puede demandar tiempo su estudio (Figura 9 y 10). El segundo, consiste en evaluar la FED a nivel de la zona de aposición 


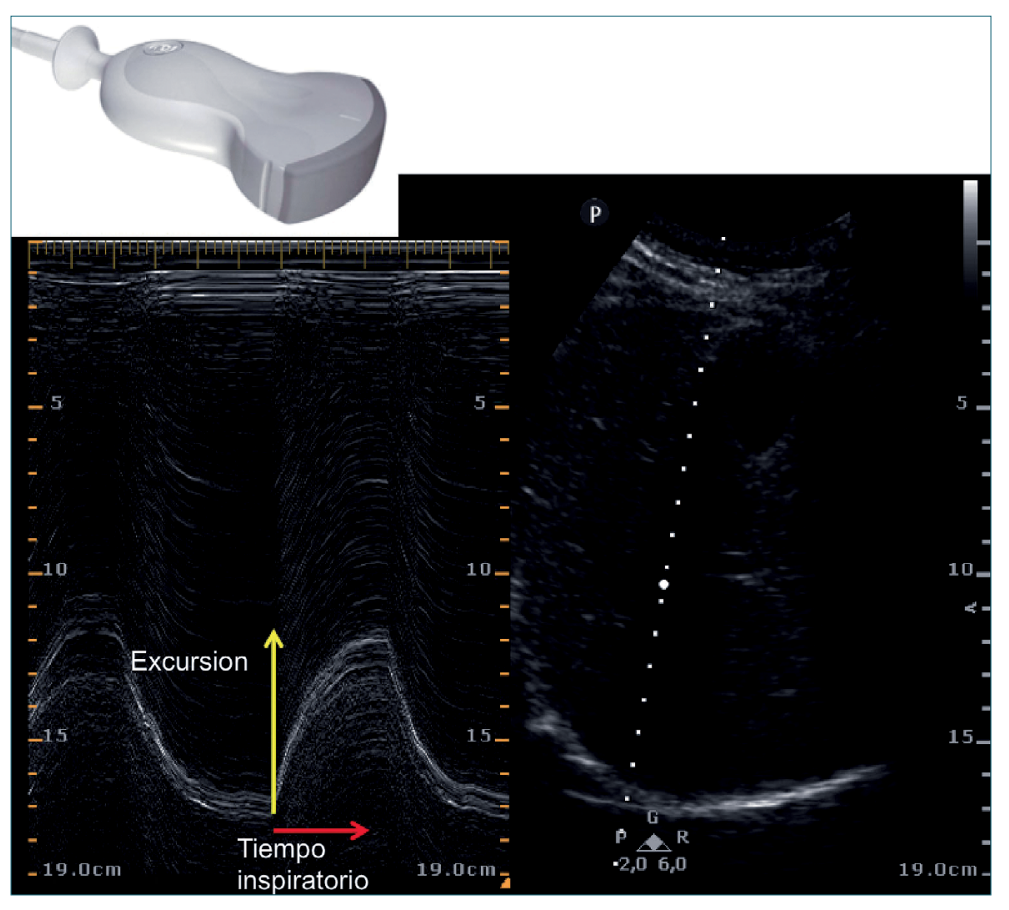

Figura 8. Estudio de la velocidad de contracción diafragmática utilizando modo M. La velocidad de contracción diafragmática esta representada por el cociente entre la Excursión inspiratoria (cm) y el Tiempo inspiratorio (seg).

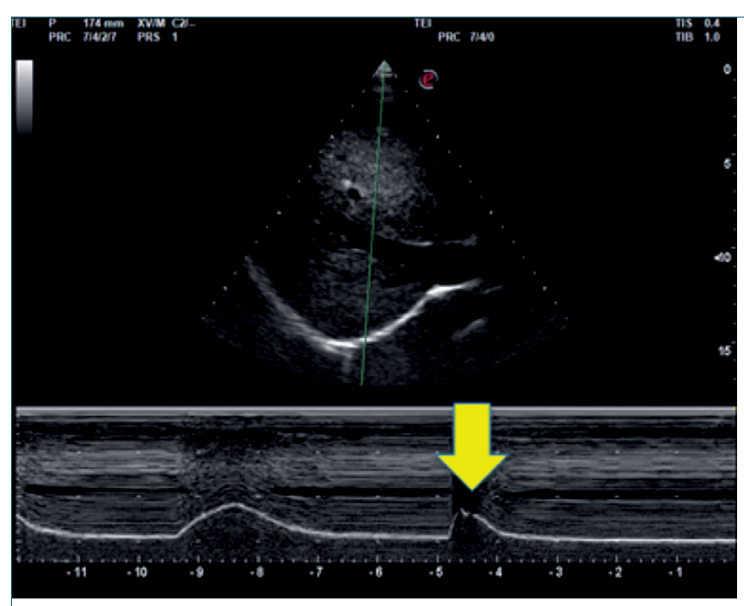

\section{Movimiento caudal del diafragma} durante la inspiración

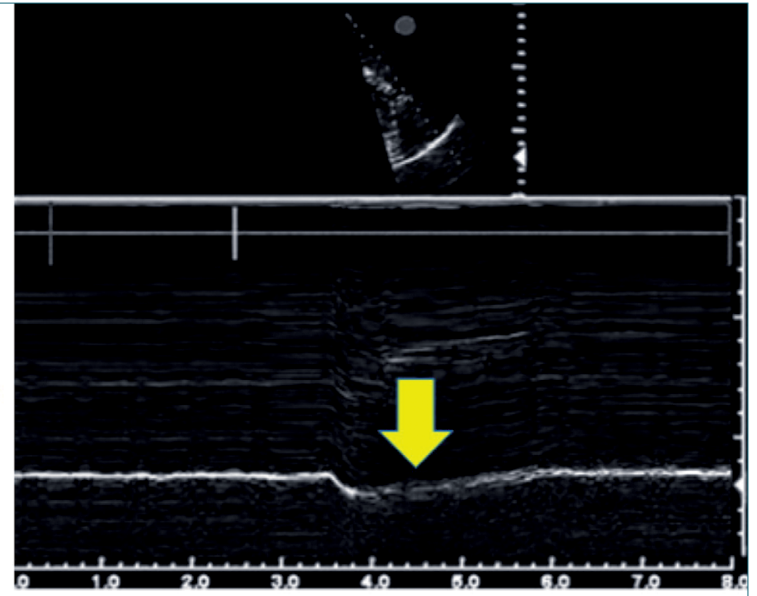

\section{Movimiento craneal del diafragma durante la inspiración}

Figura 9. Evaluación de la excursión diafragmática con sonda sectorial 2-5 MHz, utilizando el abordaje subcostal anterior. A la izquierda se observa hemidiafragma derecho en modo $\mathrm{M}$, con movimiento caudal del hemidiafragma hacia el transductor durante la maniobra de sniffing. A la derecha se evidencia parálisis diafragmática luego de realizar un bloqueo interescalénico, que genera un movimiento paradojal durante la maniobra de sniffing.

del diafragma en la caja torácica utilizando una sonda lineal de alta frecuencia[5],[9],[29], esta metodología de evaluación es más rápida y utiliza la misma sonda con que se realizó el bloqueo regional (Figura 11). Sin embargo, aún no se han reportado datos que comparen cuál de estos dos métodos es mas efectivo[31]. 

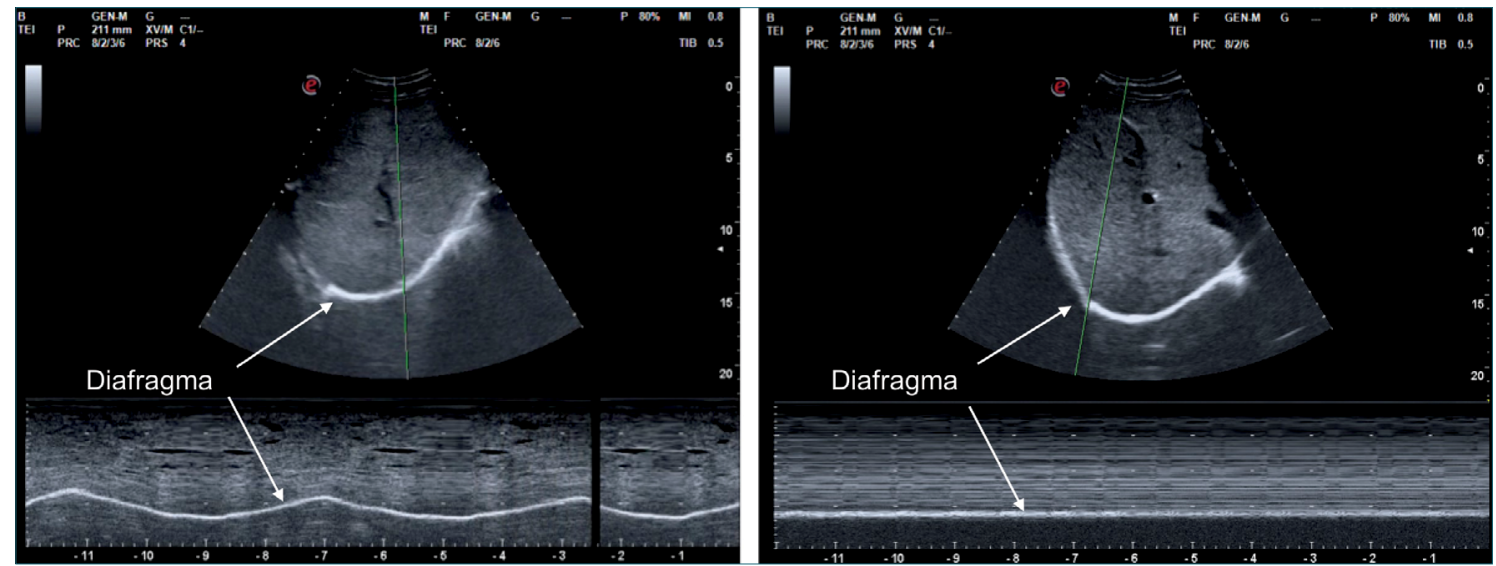

Figura 10. Evaluación de la excursión del hemidiafragma derecho con sonda convex 2-6 MHz. A la izquierda se observa movimiento caudal del hemidiafragma derecho, hacia el transductor en modo M. A la derecha se evidencia ausencia de movimiento del hemidiafragma debida a parálisis diafragmática.

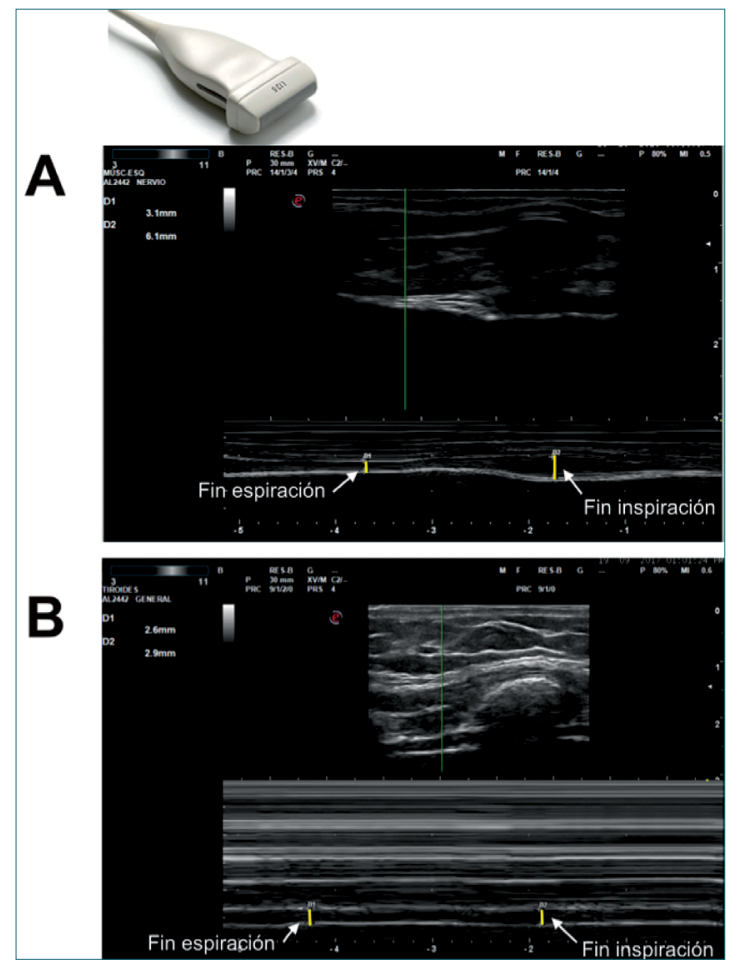

Figura 11. Medición de la fracción de engrosamiento utilizando sonda lineal 6-13 MHz a nivel de la zona de aposición, en modo B. Arriba se observa un paciente con reserva contráctil conservada, engrosamiento del hemidiafragma al fin de la inspiración y disminución del mismo al fin de espiración. Abajo deterioro en la fracción de engrosamiento debida a paresia diafragmática.

\section{2- Disfunción diafragmática en el paciente post- quirúrgico}

La disfunción diafragmática constituye uno de los factores determinantes en la patogénesis de las complicaciones pulmonares post-operatorias asociada a cirugías de tórax y abdomen superior. El deterioro en la reserva contráctil del diafragma puede prolongar la necesidad de asistencia ventilatoria mecánica y a la vez retrasar o conducir al fracaso del periodo de weaning.

Kim y colaboradores estudiaron en pacientes programados para hepatectomía la amplitud de excursión diafragmática utilizando modo $\mathrm{M}$ durante el pre y post-operatorio. Los autores encontraron durante el postoperatorio una correlación lineal entre la amplitud inspiratoria diafragmática y la capacidad vital medida a través de espirometría. El valor de corte más adecuado de amplitud diafragmática inspiratoria que detecta el 30 y $50 \%$ de disminución de la capacidad vital en el post-operatorio fue de $36 \mathrm{~mm}$ y $24 \mathrm{~mm}$ respectivamente. La sensibilidad y especificidad para detectar con ultrasonido una disminución del 30\% de la capacidad vital fue de 94 y $76 \%$, y del $81 \%$ y $91 \%$ para evidenciar una disminución del 50\%[32].

En pacientes sometidos a colecistectomía tanto por vía videolaparoscópica como convencional el examen sonográfico del diafragma en modo $\mathrm{M}$ durante el post-operatorio puede mostrar una disminución en la excursión inspiratoria durante la respiración normal y profunda[31]. Existe evidencia que sugiere que el deterioro diafragmático luego de una cirugía abdominal superior se puede explicar por la inhibición refleja 
de la actividad eferente del nervio frénico debido a la irritación de las aferencias esplácnicas, mas que por fallo contráctil o trauma quirúrgico de la pared abdominal[34],[35].

La injuria del nervio frénico puede ocurrir en el post-operatorio de cirugía cardiaca generando paresia o parálisis del hemidiafragma ipsilateral. Lerolle y su grupo estudiaron la excursión diafragmática bilateral en esta población de pacientes, identificando como parámetro funcional de performance diafragmática la excursión máxima, reportando que aquellos pacientes con parálisis diafragmática unilateral que presentaban una excursión máxima del hemidiafragma contralateral $>25 \mathrm{~mm}$ tuvieron un periodo de weaning exitoso sin prolongar el periodo de ventilación mecánica. Sin embargo, una excursión máxima $<25$ mm fue indicativa de paresia o parálisis diafragmática bilateral. Dicha disfunción diafragmática severa se asocio a mayor tiempo de requerimiento de ventilación mecánica y estadía en cuidados intensivos[36]. Este estudio evidencia la necesidad de examinar ambos hemidiafragmas en el post-operatorio de cirugía cardiaca debido a que estos pacientes se encuentran expuestos a la injuria del nervio frénico. En ocasiones durante el postoperatorio de cirugía cardiaca suele ser dificultoso examinar el diafragma utilizando la ventana subcostal debido a la mala ventana acústica o la presencia de apósitos; por lo que una alternativa es posicionar el transductor a nivel de la línea axilar media.

\section{3- Evaluación de la función diafragmática en el paciente crítico}

La evaluación del engrosamiento diafragmático a nivel intercostal en la "zona de aposición", constituye el mejor abordaje para evaluar la disfunción y atrofia diafragmática en el paciente en ventilación mecánica, ya sea de manera asistida o controlada. Los valores normales de engrosamiento a nivel de la capacidad residual funcional son 1,8 a $3 \mathrm{~mm}$. El engrosamiento diafragmático se incrementa en forma lineal hasta un $54 \%$ a medida que el volumen pulmonar aumenta desde el volumen residual a la capacidad pulmonar total[37]. Goligher y su grupo en un estudio multicéntrico, reportaron que en los primeros 8 días de ventilación mecánica el $44 \%$ de los pacientes presentaron una disminución del $10 \%$ en el engrosamiento diafragmático. En este grupo de pacientes se observo además una baja contractilidad diafragmática y un elevado "driving pressure" en los parámetros respiratorios. Los autores concluyen que la presión soporte debe ser titulada para mantener y estimular la contractilidad diafragmática evaluada a través de US[38]. De igual manera, Kim y su grupo demostraron que un
29\% de los pacientes en ventilación mecánica, sin patología diafragmática previa, presentaron disfunción diafragmática, la cual se asocio a un mayor requerimiento de asistencia ventilatoria, tiempos de weaning mas prolongados y fracaso en el weaning comparado con aquellos pacientes que no presentaron disfunción diafragmática[39].

Matamis y su grupo han reportado el rol de la evaluación sonográfica del diafragma durante la ventilación asistida. Como lo hemos mencionado previamente, es importante destacar que cuando el paciente se encuentra en una modalidad asistida existen dos fuerzas: una fuerza activa que es la contracción muscular propia del paciente y otra es el desplazamiento pasivo generado por la presión positiva externa. De tal manera que no es posible distinguir que fuerza corresponde a cada componente. Por consiguiente, si queremos evaluar la excursión diafragmática en un paciente bajo asistencia mecánica es necesario desvincularlo transitoriamente del respirador. A través de un registro en modo $\mathrm{M}$ de la excursión diafragmática y la curva de presión en via aérea es posible optimizar la sincronización entre el esfuerzo del paciente durante la ventilación asistida y el respirador[5].

\section{4- Disfunción diafragmática durante el weaning de la ventilación mecánica}

Numerosos estudios han destacado el rol de la evaluación sonográfica del diafragma en el proceso de weaning. Kim y colaboradores estudiaron la excursión diafragmática utilizando modo $\mathrm{M}$ durante el proceso de weaning, estableciendo como parámetro de disfunción diafragmática una excursión $<10 \mathrm{~mm}$ o un movimiento paradojal, para cualquiera de ambos hemidiafragmas. Reportaron una prevalencia de disfunción diafragmática del 29\%. Dicha disfunción se asocio con periodos mas prolongados de weaning, mayor incidencia de reintubación y requerimiento de ventilación mecánica[39]. De esta manera el US permite identificar pacientes con riesgo de weaning dificultoso.

Mariani y su grupo estudiaron la excursión diafragmática en pacientes deterioro en la cinética de ambos hemidiafragmas asociado a ventilación mecánica prolongada, dichos pacientes presentaron una excursión inspiratoria $<11 \mathrm{~mm}$ y evidenciaron una mejoría progresiva en la excursión en el $71 \%$ de los pacientes[40]. El examen sonográfico del diafragma no solo permite realizar el diagnostico de disfunción diafragmática sino también posee un rol clave en la monitorizar su recuperación.

Ferrari y colaboradores estudio el índice predictivo de weaning después de una prueba de ventilación es- 
pontanea, reportando que una FED $\geq 36 \%$ se asocio a un weaning exitoso con una sensibilidad del $82 \%$ y una especificidad del $88 \%$, un valor predictivo positivo de $92 \%$ y un valor predictivo negativo de $75 \%$ para el éxito o falla de la discontinuación de la ventilación mecánica a las 48 horas[41]. Este grupo realizo la evaluación de la FED a nivel de la zona de aposición, posicionando el transductor lineal a nivel del 8-9 espacio intercostal entre la línea axilar anterior y media; realizando dos mediciones: a capacidad pulmonar total y luego a volumen residual. Utilizaron modo B debido a que esta técnica aporta mayor definición anatómica del musculo y sus estructuras adyacentes junto con una visión más panorámica en comparación al modo M[41]. Di Nino realizo un estudio similar en 63 pacientes, reportando que una FED $\geq 30 \%$ se asocio con un valor predictivo positivo de $91 \%$ y un valor predictivo negativo de $63 \%$ para un weaning exitoso[42].

En la Tabla 2 se describen las principales mediciones utilizadas en el paciente bajo soporte ventilatorio durante la ventilación mecánica.

\section{5- Función diafragmática en el paciente EPOC}

Numerosos estudios han demostrado la utilidad del US en el estudio del diafragma en el paciente EPOC. Zanforlin y su grupo describieron un índice que podría indirectamente detectar un patrón obstructivo de la vía aérea a través del cálculo del índice de obstrucción en modo M (IOM). Estableciendo un valor de corte para sospecha de patrón espirométrico obstructivo $<77$ con un valor predictivo positivo de 95,5\%[43]. El IOM representa la velocidad de relajación diafragmática y se calcula (Figura 12):
IOM \% = Excursión diafragmática espiratoria forzada en el $1^{\circ}$ segundo

Excursión diafragmática espiratoria máxima

Este índice podría ser una alternativa a la evaluación de la curva volumen/tiempo obtenida por espirometría, considerada técnica de elección, la cual analiza la relación entre el volumen espiratorio forzado del $1^{\circ}$ segundo $\left(\mathrm{FEV}_{1}\right)$ y la capacidad pulmonar.

Dentro de las limitaciones de esta técnica encontramos la mala ventana acústica, especialmente en pacientes obesos, heridas que impidan el adecuado posicionamiento de la sonda o la presencia de enfisema subcutáneo.

\section{Conclusiones}

En resumen, podemos decir que el examen sonográfico del diafragma constituye una nueva herramienta para el anestesiólogo, permite evaluar la función diafragmática del paciente quirúrgico durante el peri-operatorio de manera dinámica, no invasiva y a la cabecera del paciente.

EI US del diafragma, constituye un método reproducible y con poca variabilidad inter-observador, puede ser repetido a lo largo del tiempo permitiendo una monitorización dinámica de la recuperación de la fuerza contráctil en el paciente con disfunción diafragmática. Probablemente la evaluación sonográfica del engrosamiento diafragmático a nivel de la zona de aposición utilizando la sonda lineal constituye el abordaje mas atractivo para los anestesiólogos a la hora de evaluar una disfunción durante el peri-operatorio, debido a que técnicamente es mas rápido y simple

\begin{tabular}{|c|c|c|c|c|}
\hline Medición & Tipo de sonda & Valor & Implicancia clínica & Referencia \\
\hline E diafragmática & convex & $<11 \mathrm{~mm}$ & $\begin{array}{l}\text { Aumento probabilidad fallo } \\
\text { weaning }\end{array}$ & Mariani et al.[28] \\
\hline $\begin{array}{l}\text { E diafragmática de- } \\
\text { recha/izquierda }\end{array}$ & convex & $\begin{array}{l}\text { Ausencia bilateral } \\
\text { de } \mathrm{E}\end{array}$ & $\begin{array}{l}\text { Aumento probabilidad fallo } \\
\text { weaning }\end{array}$ & Lerolle et al.[24] \\
\hline $\begin{array}{l}\text { Mejor E diafrag- } \\
\text { mática derecha/iz- } \\
\text { quierda }\end{array}$ & convex & $>25 \mathrm{~mm}$ & $\begin{array}{l}\text { Aumento probabilidad éxito } \\
\text { weaning }\end{array}$ & Lerolle et al.[24] \\
\hline FED & lineal & $\geq 36 \%$ & $\begin{array}{l}\text { Aumento probabilidad éxito } \\
\text { weaning }\end{array}$ & Ferrari et al.[29] \\
\hline FED & lineal & $\geq 30 \%$ & $\begin{array}{l}\text { Aumento probabilidad éxito } \\
\text { weaning }\end{array}$ & Di Nino et al. [30] \\
\hline
\end{tabular}

E: excursión. FED: Fracción de engrosamiento diafragmático. 


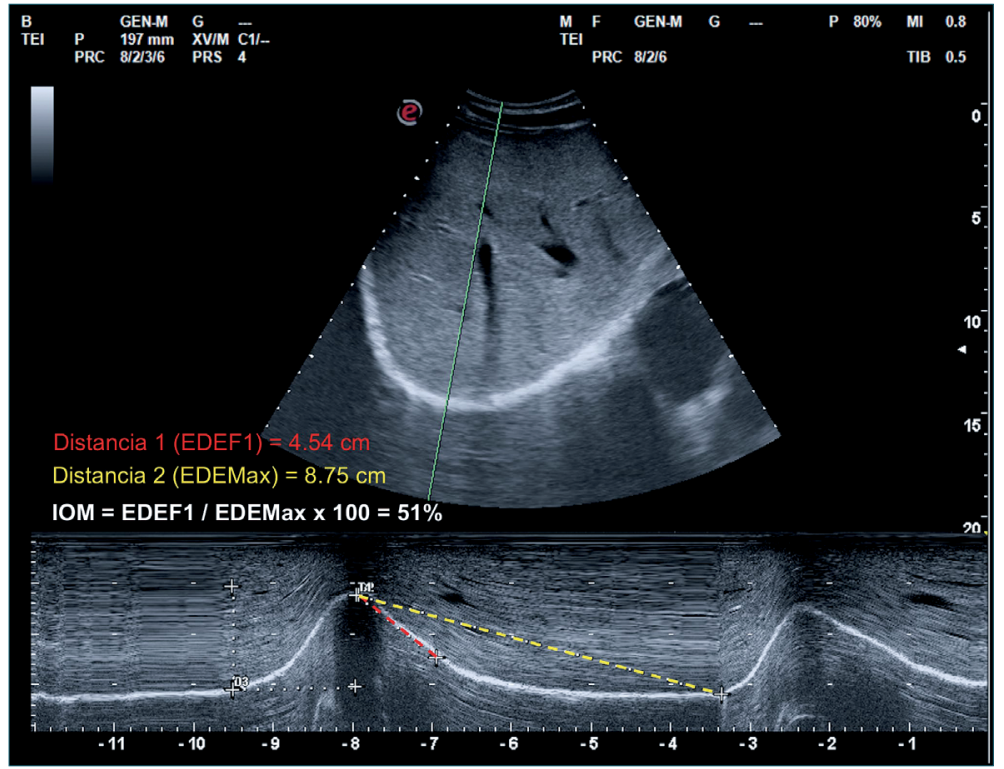

Figura 12. Cálculo del índice de obstrucción en modo $\mathrm{M}$ (IOM) durante una espiración forzada en un paciente EPOC. IOM = Excursión Diafragmática Espiratoria máxima $\left[\mathrm{EDE}_{\text {máx }}\right]$ / Excursión Diafragmática Espiratoria Forzada del $1^{\circ}$ segundo $\left.\left[\mathrm{FEDE}_{1}\right]\right)=51 \%$.

de realizar, requiere la misma sonda que utilizamos para abordar los bloqueos loco-regionales o punciones vasculares ecoguiadas.

La evaluación sonográfica peri-operatoria del diafragma debería ser utilizada de manera integrada al examen sonográfico del pulmón ante un paciente con fallo respiratorio agudo, permitiendo de esta manera el diagnostico de patologías pulmonares como derrame pleural o consolidaciones que pueden generar una disfunción diafragmática como así también evidenciar disfunciones previas. De esta manera podemos optimizar nuestra estrategia anestésica en pacientes críticos con riesgo a presentar complicaciones respiratorias en el post-operatorio.

Son necesarios estudios que evalúen el rol y limitaciones en la monitorización del paciente critico.

\section{Referencias}

1. Al-Bilbeisi F, McCool FD.

Diaphragm recruitment during nonrespiratory activi- ties. Am J Respir Crit Care Med 2000; 162:456-9.

2. Loring $\mathrm{SH}$, Mead J. Action of the dia- phragm on the rib cage inferred from a forcebalance analysis. J Appl Physiol 1982;53:756-60.

3. Haber K, Asher M, Freimanis AK: Ultrasonographic evaluation of diaphragmatic motion in intraabdominal diseases. Radiology 1975, 114:141-4.

4. Boussuges A, Gole Y, Blanc P. Diaphragmatic motion studied by M-mode ultrasonography. Chest 2009; 135:391-400.

5. Matamis D, Soilmezi

\section{E, Tsagourias M, et al.} Sonographic evaluation of the diaphragm in critically ill patients. Technique and clinical applications. Intensive Care Med 2013;39:801-810. DOI 10.1007/ s00134-013-2823-1.

6. Gerscovich EO, Cronan M, Mc Gahan JP, Jain K, Jones D, Mc Donald C. Ultrasonographic evaluation of diaphragmatic Motion. J Ultrasound Med 2001; 20: 597-604.

7. Lloyd T, Tang YM, Benson MD, et al: Diaphragmatic paralysis: The use of $\mathrm{M}$ mode ultrasound for diagnosis in adults. Spinal Cord 2006; 44:505-8.

8. Alexander C. Diaphragm movements and the diagnosis of diaphragmatic paralysis. Clin Radiol. 1966; 17(1):79-83.

9. Tsui JJ, Tsui BC. A novel systematic $A B C$ approach to diaphragmatic evaluation (ABCDE). Can J Anaesth. 2016;63:636-7.

10. Tsui BC, Tsui J. ABC diaphragmatic evaluation for neonates. Paediatr Anaesth. 2016;26:768-769.

11. Jiang JRm Tsai TH, Jerng JS, Yu CJ, Wu HD, Yang PC: Ultrasonographic evaluation of liver/spleen movements and 
extubation outcome. Chest 2004, 126:179-185.

12. Goligher EC, Laghi F, Detsky $\mathrm{ME}$, et al. Measuring diaphragm thickness with ultrasound in mechanically ventilated patients: feasibility, reproducibility and validity. Impact of inspiratory effort. Intensive Care Med 2015; 41:642-9.

13. Umbrello $M$, Formenti $P$, Longhi $D$, et al. Diaphragm ultrasound as indicator of respiratory effort in critically ill patients undergoing assisted mechanical ventilation: a pilot study. Critical Care 2015; 19:161.

14. Urmay WF, Talts KH, Sharrock NE: One hundred percent incidence of hemidiaphragmatic paresis associated with interscalene brachial plexus anesthesia as diagnosed by ultrasonography. Anesth Analg 1991;72:498-503.

15. Urmey WF, Gloeggler PJ. Pulmonary function changes during interscalene brachial plexus block: effects of decreasing local anesthetic injection volume. Reg Anesth. 1993;18:244-9.

16. Sala-Blanch $X$, Lázaro JR, Correa J, Gómez-Fernandez M. Phrenic nerve block caused by interscalene brachial plexus block: effects of digital pressure and a low volume of local anesthetic. Reg Anesth Pain Med. 1999;24:231-5.

17. Riazi S, Carmichael N, Awad I, Holtby RM, McCartney CJ. Effect of local anaesthetic volume (20 vs $5 \mathrm{ml}$ ) on the ef cacy and respiratory consequences of ultrasound-guided interscalene brachial plexus block. Br J Anaesth. 2008;101:549-556.

18. Fujimura $\mathrm{N}$, Namba $\mathrm{H}$, Tsunoda K, Kawamata T, Taki K, Igarasi M, Namiki A: Effect of hemidiaphragmatic paresis caused by interscalene brachial plexus block on breathing pattern, chest wall mechanics, and arterial blood gases. Anesth Analg 1995; 81:962-6.

19. Wong AK, Keeney LG, Chen L, Williams R, Liu J, Elkassabany NM: Effect of local anesthetic concentration $(0.2 \%$ vs $0.1 \%$ ropivacaine) on pulmonary function, and analgesia after ultrasound-guided interscalene brachial plexus block: A randomized controlled study. Pain Med 2016; 17:2397-403.

20. Thackeray EM, Swenson JD, Gertsch MC, Phillips KM, Steele JW, Burks RT, Tashjian RZ, Greis PE: Diaphragm function after interscalene brachial plexus block: A double-blind, randomized comparison of $0.25 \%$ and $0.125 \%$ bupivacaine. J Shoulder Elbow Surg 2013; 22:381-6

21. Pere $P$, Pitkänen $M$, Rosenberg $\mathrm{PH}$, Björkenheim JM, Linden $\mathrm{H}$, Salorinne $Y$, Tuominen $M$ : Effect of continuous interscalene brachial plexus block on diaphragm motion and on ventilatory function. Acta Anaesthesiol Scand 1992; 36:537.

22. Smith MP, Tetzlaff JE, Brems $\mathrm{JJ}$ : Asymptomatic profound oxy-hemoglobin desaturation following interscalene block in a geriatric patient. Reg Anesth Pain Med 1998; 23:210-3.

23. Altintas F, Gumus F, Kaya G, Mihmanli I, Kantarci F, Kaynak K, Cansever MS: Interscalene brachial plexus block with bupivacaine and ropivacaine in patients with chronic renal failure: Diaphragmatic excursion and pulmonary function changes. Anesth Analg 2005; 100:1166-71.

24. Haskins SC, Tsui BC, Nejim JA, Wu CL, Boublik J. Ultrasound for the regional anesthesiologist and acute pain specialist. Reg Anesth
Pain Med 2017;42:289-298.

25. Al-Kaisy AA, Chan VW, Perlas A: Respiratory effects of low-dose bupivacaine interscalene block. $\mathrm{Br} J$ Anaesth 1999; 82:217-20.

26. El-Boghdadly K, Chin KJ, Chan VWS. Phrenic nerve palsy and regional anesthesia for shoulder surgery. Anesthesiology 2017;127:173-91.

27. Boon AJ, Sekiguchi $H$, Harper CJ, Strommen JA, Ghahfarokhi LS, Watson JC, Sorenson EJ: Sensitivity and specificity of diagnostic ultrasound in the diagnosis of phrenic neuropathy. Neurology 2014; 83:1264-70.

28. Houston JG, Fleet M, Cowan MD, McMillan NC. Comparison of ultrasound with fluoroscopy in the assessment of suspected hemidiaphragmatic movement abnormality. Clin Radiol 1995;50:95-8.

29. Testa A, Soldati G, Giannuzzi $\mathrm{R}$, et al. Ultrasound M-mode assessment of diaphragmatic kinetics by anterior transverse scanning in healthy subjects. Ultrasound Med Biol. 2011;37:44-52.

30. Zambon M, Cabrini L, Beccaria $\mathrm{P}$, Zangrillo A, Colombo S. Ultrasound in critically ill patients: focus on diaphragm. Intensive Care Med 2013; 39:986.

31. Zambon M, Greco M, Bocchino S, Cabrini L, Beccaria PF, Zangrillo

A. Assessment of diaphragmatic dysfunction in the critically ill patient with ultrasound: a systematic review. Intensive Care Med 2017;43:29-38.

32. Kim SH, Na S, Choi JS, Na $\mathrm{SH}$, et al. An evaluation of diaphragmatic movement by M-mode sonography as a predictor of pulmonary dysfunction after upper abdominal surgery. Anesth Analg 2010;110:1349-54.

33. Ayoub J, Cohendy R, Prioux J, 
Ahmaidi S, Bourgeois JM, Dauzat

M, Ramonatxo M, Prefaut C.

Diaphragm movement before and after cholecystectomy: a sonographic study. Anesth Analg 2001;92:755-61

34. Ford GT, Whitelaw WA, Rosental TW, et al. Diaphragm function after abdominal surgery in humans. Am Rev Respir Dis 1983;127:431-6.

35. Dureuil B, Viires N, Cantineau JP, et al. Diaphragmatic contractility after upper abdominal surgery. J Appl Physiol 1986;61: 1775-80.

36. Lerolle N, Guérot E, Dimassi $S$, et al. Ultrasonographic diagnostic criterion for severe diaphragmatic dysfunction after cardiac surgery. Chest 2009; 135(2): 401-7.
37. Cohn D, Benditt JO, Eveloff S, Mc Cool D. Diaphragm thickening during inspiration. J Appl Physiol 1997; 83: 291-6.

38. Goligher EC, Fan E, Herridge MS, et al. Evolution of diaphragm thickness during mechanical ventilation. Impact of inspiratory effort. Am J Respir Crit Care Med 2015; 192:1080-8.

39. Kim WY, Suh HJ, Hong SB, et al. Diaphragm dysfunction assessed by ultrasonography: influence on weaning from mechanical ventilation. Crit Care Med 2011;39:2627-30.

40. Mariani LF, Bedel J, Gros A, Lerolle $\mathrm{N}$, et al. Ultrasonography for screening and follow-up of diaphragmatic dysfunction in the ICU: a pilot study. J Intensive
Care Med. 2016;31:338-43.

41. Ferrari G, De Filippi G, Elia F, Panero F, Volpicelli G, Aprà F. Diaphragm ultrasound as a new index of discontinuation from mechanical ventilation. Crit Ultrasound J 2014;8:8.

42. DiNino E, Gartman EJ, Sethi JM, McCool FD. Diaphragm ultrasound as a predictor of successful extubation from mechanical ventilation. Thorax 2014;69:423-7.

43. Zanforlin A, Smargiassi A, Inchingolo $R$, et al. Ultrasound analysis of diaphragm kinetics and the diagnosis of airway obstruction: the role of the M-mode index of obstruction. Ultrasound Med Biol 2014; 40(6):1065-71. 